\title{
Germanica
}

\section{Un Don Quichotte contre la Laideur : Henry Van de Velde}

Ein Don Quichotte gegen die Häßlichkeit: Henry Van de Velde

\section{Alexandre Kostka}

\section{OpenEdition}

\section{Journals}

Édition électronique

URL : http://journals.openedition.org/germanica/443

DOI : 10.4000/germanica.443

ISSN : 2107-0784

Éditeur

Université de Lille

Édition imprimée

Date de publication : 1 décembre 2005

Pagination : 51-66

ISBN : 2-913857-16-7

ISSN : 0984-2632

Référence électronique

Alexandre Kostka, «Un Don Quichotte contre la Laideur : Henry Van de Velde », Germanica [En ligne],

37 | 2005, mis en ligne le 07 janvier 2010, consulté le 06 octobre 2020. URL : http://

journals.openedition.org/germanica/443 ; DOI : https://doi.org/10.4000/germanica.443

Ce document a été généré automatiquement le 6 octobre 2020.

(c) Tous droits réservés 


\title{
Un Don Quichotte contre la Laideur : Henry Van de Velde ${ }^{1}$
}

Ein Don Quichotte gegen die Häßlichkeit: Henry Van de Velde

\author{
Alexandre Kostka
}

1 L'architecte et décorateur belge Henry Van de Velde (1863-1957) est reconnu aujourd'hui comme l'un des créateurs majeurs de l'art nouveau, aux côtés de Peter Behrens ou de Paul Guimard. Van de Velde a connu une période de purgatoire exceptionnellement longue, due en partie au fait que l'importante part allemande de sa carrière rendait difficile son intégration dans un patrimoine national belge ; le fait qu'il ait assumé durant la Seconde Guerre mondiale des fonctions officielles dans la Belgique de l'occupation n'a pas peu contribué à ternir son image ${ }^{2}$. Après sa mort, seulement une poignée de fidèles continua à lui porter hommage ${ }^{3}$. Ce n'est que depuis le milieu des années quatre-vingt que d'importantes publications et expositions majeures ont commencé à présenter au grand public la carrière d'un artiste hors du commun, qui fut aussi un médiateur de premier plan entre la France et l'Allemagne ${ }^{4}$.

2 Van de Velde se concevait comme un champion de la Beauté (qu'il écrit toujours, comme la Laideur, avec une majuscule), voire comme un ange armé d'une religion de l'art, qui visait à transformer l'ici-bas en un espace d'absolue harmonie. La laideur n'a pas sa place dans ce monde: elle incarne le Mal absolu, le vice, la déchéance de l'humanité. On reconnaît là un aspect de l'idéologie du Gesamtkunstwerk, de l'œuvre d'art total, qui hante toute l'Europe de la fin du $\mathrm{xIx}^{\mathrm{e}}$ siècle, et particulièrement l'Allemagne. Mais chez Van de Velde, il s'agit d'une vision coercitive, voire totalitaire du Beau intégral, qui va jusqu'à habiller les femmes d'une tenue adaptée au papier mural, tandis que les hommes devaient tous être habillés en frac! Même les pâtissiers sont investis d'une responsabilité morale à réfléchir sur la bonne forme qu'ils doivent donner aux petits-pains, autant que les coiffeurs qui doivent penser à la bonne forme des moustaches de leurs clients... ${ }^{5}$

3 Un des traits qui singularise son œuvre est son activité de théoricien, activité qui a constamment sous-tendu, tout particulièrement pendant sa première période d'activité, laquelle s'étend des années 1890 à la veille de la Première Guerre mondiale, 
sa création artistique. Notre contribution vise justement à porter un regard neuf sur l'œuvre théorique de Van de Velde, à travers le prisme d'une question centrale - celle de l'opposition absolue entre Beauté et Laideur. Notre point de vue est que la réflexion de Van de Velde, loin de ne constituer qu'une stratégie de légitimation d'un artiste autodidacte (ce qu'elle est assurément aussi...), est absolument centrale pour comprendre sa démarche créatrice ${ }^{6}$. Elle n'est pas non plus seulement l'élucubration d'un individualiste inclassable qui ne parle que pour lui-même : elle reflète au contraire les préoccupations de son époque, qui accouchait difficilement de la Modernité que nous connaissons aujourd'hui, et qui a fait de la « laideur » une des catégories centrales pour comprendre son identité dispersée. Il parait facile de dénoncer en Van de Velde un nostalgique d'un ordre d'autrefois, où "harmonie ", «mesure " et "beauté " règnent en maitre, voire, avec Adolf Loos, comme le représentant d'une moralité hypocrite qui habille le capitalisme consumériste d'un ornement pour mieux en dissimuler la véritable nature ${ }^{7}$. Or, son cas est bien plus complexe, et fait rentrer celui qui s'intéresse à Van de Velde sans préjugés dans le domaine d'une pensée esthétique et utopiste, au sein de laquelle morale et création artistique vont de pair.

\section{Comment la Laideur vint au monde}

4 Si l'on rassemble un grand nombre de remarques provenant d'œuvres théoriques diverses, qui s'étendent de ses débuts dans le milieu des symbolistes et néoimpressionnistes bruxellois à la grande crise des années vingt après son émigration forcée d'Allemagne et l'impossibilité du retour en Belgique, on peut esquisser une théorie esthétique qui conçoit la laideur comme la négation pure et simple de la raison et de la morale, voire de l'humanité même ${ }^{8}$. Pour l'athéiste et l'anarchiste Van de Velde, la Laideur se situe exactement à l'endroit où la doctrine chrétienne plaçait le diable et le vice, et qu'il faut éradiquer avec la dernière fermeté. À un moment où Baudelaire et son esthétique de la laideur s'était imposé depuis longtemps dans les avant-gardes que le jeune Van de Velde fréquentait à Bruxelles, une telle position ne peut que surprendre, et appelle quelques éclaircissements.

5 Grâce aux recherches récentes, et notamment grâce à l'analyse de la bibliothèque de Van de Velde conservée aujourd'hui à Bruxelles à la Cambre - École Nationale Supérieure des Arts Visuels, nous savons que la réflexion théorique de Van de Velde était nourrie par un grand nombre de sources, parmi lesquelles les travaux d'anthropologie portant sur l'origine de l'humanité et la psychologie contemporaine (Wundt, Chevreuil, Helmholtz...) occupaient une place importante9. Comme de nombreux autres théoriciens qui l'avaient précédé (Laugier, Semper...) Van de Velde voulait remonter vers les origines supposées de l'humanité pour comprendre l'élan primitif de la création artistique. Contrairement par exemple à Semper, qui avait cerné les origines de l'art dans l'imitation de certaines formes immédiates de l'ornement (comme les couvertures de la maison, des trames de tissage, des formes trouvées dans la nature...), Van de Velde fait dériver l'art de l'activité rythmique de l'homme, du geste, voire de la danse ${ }^{10}$.

6 Selon Van de Velde, l'art met en œuvre un certain nombre de constantes anthropologiques (notamment de la perception et du rythme), qui sont modifiées avec le temps et en fonction des environnements. Nous nous trouvons donc dans une perspective radicalement matérialiste, qui omet cependant de mentionner les 
concepts-clé (race, temps, milieu) d'une esthétique positiviste qui voyait en Taine son fondateur ${ }^{11}$. La raison est sans doute que selon Taine et les tenants de la critique positiviste, il ne pouvait y avoir d'art que de son époque : or l'ambition de Van de Velde était de créer un art universel, obéissant de manière rationnelle à certains préceptes immuables du Beau qu'il se faisait fort d'avoir découvert de manière spontanée, et qui pouvaient constituer les «fondements " d'un «Style Nouveau » qui serait de tous les temps ${ }^{12}$. Plus tard, des scientifiques viendraient pour donner une explication rationnelle aux créations que lui, Van de Velde, produisait intuitivement ${ }^{13}$.

7 La Beauté (nous reprenons l'orthographe de Van de Velde) résulte donc de l'accord de l'homme avec ses propres facultés motrices, sensorielles et "psychologiques $»^{14}$. Inversement, la Laideur signifie un désaccord de l'homme avec lui-même, une forme particulièrement pernicieuse d'aliénation; le manque d'harmonie entre les facultés génère non seulement un déplaisir physique, mais encore un manque d'orientation dans le monde qui l'entoure et une dysharmonie des relations entre les hommes. La laideur attaque donc l'homme non seulement de l'extérieur, mais aussi - et surtout - de l'intérieur. Bref, la laideur est selon Van de Velde le chancre du monde moderne, dont il faut cerner l'origine pour pouvoir le traiter à l'instar d'un médecin guérissant un mal redoutable.

\section{La Révolution française comme percée de la Laideur dans le monde moderne}

8 Dans l'évolution de l'art européen, Van de Velde décèle deux ruptures majeures :

- la première rupture est celle de la Renaissance, qui aurait dissocié les beaux-arts de l'art décoratif, et détruit l'unité médiévale des $\operatorname{arts}^{15}$. L'affranchissement de l'œuvre d'art de son environnement par le tableau de chevalet aurait détruit l'unité gothique entre l'habitat de l'homme et l'usage public de l'art: dissocié de son emplacement, le tableau serait tombé au rang d'objet de plaisir, voire de commerce. Les arts décoratifs, désormais considérés comme "mineurs", n'auraient plus pu jouer leur rôle de cadre harmonieux destiné à magnifier la vie des hommes et seraient peu à peu tombé entre les mains d'artisans moins inspirés et routiniers. Pourtant, une clientèle aristocratique pourvue du « bon goût » aurait réussi créer un cadre adapté à ses besoins, comme ce fut le cas avec le classicisme français.

- La Révolution française aurait définitivement détruit la continuité de l'évolution des styles, et amorcé une "dérive» qui atteindrait son apogée dans la fabrication capitaliste d'objets en série destinés au marché ${ }^{16}$. En guillotinant les détenteurs du «bon goût » et en interdisant les corporations d'artisans, la Révolution française aurait porté le coup le plus fatal à l'art. Privé de direction, le "vaisseau de l'art européen » aurait commencé une errance au gré des vagues qui durerait jusqu'à l'arrivée de Van de Velde $^{17}$. L'aventure napoléonienne, érigeant le classicisme comme esthétique officielle, aurait été trop courte pour générer un véritable "style "; après la confusion de la période post-révolutionnaire et post-napoléonienne, l'industrialisation rapide aurait généré dans les arts décoratifs un art totalement faux par les matériaux employés et absolument laid par les formes imitées. Toute véritable création serait désormais empêchée par la loi d'airain du capitalisme de marché, qui empêcherait une véritable création de s'accomplir. La production est éclectique, chaotique, dépourvue d'unité. La laideur contemporaine, pour Van de Velde, recoupe donc largement les analyses de 
Walter Benjamin dans Berliner Kindheit um 1900 (1932): il s'agit de la cacophonie des styles accolés les uns aux autres sans raison, bref de ce qu'il est convenu d'appeler «l'historisme », par quoi il faut entendre l'absence d'un principe unifiant de la création artistique $^{18}$.

Il est cependant possible de pousser la généalogie de la Laideur encore plus loin dans le temps. La «chute» de l'humanité dans la laideur serait due au christianisme, plus précisément à la déchéance de la divinité universelle qui s'incarnerait dans un seul homme particulier : «Le dieu qui s'abaissa au niveau des hommes insinua la Laideur entre ces deux pôles d'art (= la représentation de l'Horreur ou de la Beauté, AK), - la Laideur inconnue auparavant $"^{19}$. L'anti-christianisme esthétique est un thème que Van de Velde évoque fréquemment, sans jamais l'expliciter tout à fait ${ }^{20}$. Ce point est cependant important dans la mesure où il permet à Van de Velde de postuler un art comme expression naturelle et «pure » de la vitalité humaine, s'étendant des origines primitives jusqu'à l'extrême pointe de la civilisation qu'était le monde grec présocratique. Le créateur belge rejoint là les analyses de Nietzsche, qui fut, comme il l'affirme lui-même, une référence majeure dans sa décision de se tourner vers les arts appliqués.

Van de Velde rapproche fréquemment la Laideur du «sentiment » alors que la Beauté serait proche de la "raison ${ }^{21}$. Le champ sémantique de "sentiment» (Gefühl) « sentimentalité » (sentimental), voire «fantaisie » (Phantasie; le mot n'a pas la même signification dans les deux langues) est connoté très négativement, et fréquemment rapproché de la "croyance » chrétienne. Le "sentiment » substitue à une perception claire du monde réel le domaine changeant des émotions: on reconnaît là une réminiscence des théories platoniciennes ${ }^{22}$. La "raison" (Vernunft) est seule capable d'introduire un « ordre » unifiant dans la création esthétique - toute forme du Beau est donc rationnelle (Vernunftgemässe Schönheit), car elle transfère une logique qui était à l'œuvre dans l'art du passé - par lequel il faut entendre : art grec - dans le temps présent $^{23}$. Et les temps modernes sont selon Van de Velde marqués par le sceau de la " raison ", de sorte qu'il y a une dichotomie entre ce que la société est déjà (rationnelle) et ce qu'elle croit être (sentimentalité) : là réside le «mal » qu'il s'agit d'éradiquer par une chasse systématique aux ornementations superflues ${ }^{24}$.

Avant que l'idée d'un style nouveau se fut implanté parmi nous, il était d'usage de traiter la Laideur par la Beauté. Mais le remède est inopérant! Nous proposons comme remède, la Raison. Dans le domaine de la vie pratique, rien ne devient laid qui est né d'une conception rationnelle, tandis que tout ce qui risque de devenir laid naît d'une conception sentimentale ${ }^{25}$.

11 L'historisme est l'ennemi par excellence, car il est totalement de l'ordre du « sentimental» ou encore de l'imagination (Phantasie, en allemand), alors que le style grec offre l'archétype d'une beauté « rationnelle» (vernunftgemäß), telle que Van de Velde souhaite la créer pour les temps présents. Selon Van de Velde, il suffit de décrire un meuble ou une construction historiste, en étalant l'inutilité de l'ornement dont il est affublé, pour qu'il se décompose sous l'action de la raison: "Cette expérience n'a jamais manqué de vérifier que notre cerveau est moins corrompu que nos yeux !26».

12 Le «Style nouveau » de Van de Velde prend donc le contre pied de la "dispersion » des temps modernes, et vise à introduire une unité absolue dans toutes les expressions de la vie : il s'agit, bien évidemment, d'une reprise quasi textuelle des idées de Nietzsche, qui prennent elles-mêmes leur source dans une interprétation particulière d'un 
«phantasme grec» propre au xix ${ }^{\mathrm{e}}$ finissant. C'est un point sur lequel il y aura à revenir.

\section{Perversion du laid}

13 La génération de Van de Velde, celle qui est née aux alentours de 1850 ou 1860, serait celle qui aurait été pour la première fois exposée à une laideur intégrale, et dont la génération des parents serait responsable. La constatation accablante que dresse Van de Velde est trop déchirante pour ne pas lui faire un peu de place.

Sur notre enfance pèse l'ennui morne des maisons en lesquelles nous grandîmes, où pas une chose ne possédait les qualités, les vertus qui font que les choses sont capables de dégager, autant que les êtres, un fluide de sympathie vivifiante et de confiante affection [...] Sur note âge d'homme jeune pèse l'infinie laideur des salles d'école, une laideur rongeante, comme le vice, le cœur, le cerveau, la chair ; une laideur sale comme la boue des "grandes villes ", qui colle à la chair, au cerveau, au cœur ! Et ainsi souillés, nous nous mîmes en route! La prévoyance paternelle nous avait mise en garde contre bien des maladies, mais celle de la laideur, on nous l'avait inoculée; et ses ravages sont dans nos moëlles! C'est par miracle que nous nous sommes dépêtrés ${ }^{27}$. notamment dans l'incohérence des appartements historicistes des grandes villes. Il s'agit de lieux communs que l'on retrouve aussi, comme nous l'avons dit, sous la plume de Walter Benjamin, lui-même inspiré par la lecture des ouvrages de Van de Velde et de Paul Scheerbarth. L'incohérence du monde bourgeois trouve son expression dans l'ameublement destiné seulement à impressionner les visiteurs par le faux luxe et dont la forme s'est dissociée de la fonction.

La dimension la plus intéressante cependant est celle d'une critique de la corruption morale de la laideur qui ronge « le cœur, le cerveau et la chair » comme un « vice » et «souille» les hommes jeunes comme la syphilis. La laideur est donc une véritable menace pour la société ; mais ses effets ne sont sentis que par ceux qui sont trop jeunes pour se défendre : les adultes, eux, sont tellement empoisonnés par la laideur que tout espoir de prise de conscience est vain.

17 Il faut donc que l'élan libérateur vienne d'ailleurs : ce sont les êtres qui ont conservé même à un âge adulte une sensibilité suffisamment vive pour souffrir physiquement de la laideur - les « décadents » - qui doivent accepter une «mission» de lutte contre la Laideur.

Proches du « dandy » de Baudelaire, ces êtres se barricadent derrière l'impassibilité du bon goût et d'un maintien distingué. Mais derrière le masque, ces «épicuriens de l'esprit » sont agités par la moindre sensation, la moindre disharmonie.

Ils se fixent sur les bords des mers du Nord qui sont douces et grises et ceux qui ont épuisé le charme efféminé et pervers des poèmes de la basse latinité, le poison de toutes les œuvres que Huysmans aligna dans son catalogue magnifique d'À Rebours, passent de longues heures à s'alanguir à la lecture des poètes chinois du vi siècle, à méditer les pensées de Zarathoustra, à s'éblouir au rayonnement prismatique des vers d'Herman Gorter ${ }^{28}$.

Dans l'éloignement voulu de la ville chaotique, le « décadent » peut se mettre à l'abri de la laideur du monde moderne. 
20 Les «décadents" attendent l'heure pour sauver le monde: il viendra lorsque le Nouveau monde, les Amériques vont exporter vers l'Europe un art débarrassé d'ornements inutiles : "Ils pressentent que l'Art Nouveau se bégayera par un peuple innocent et ravi et qui suivra d'amour et de soins les diverses phases de sa transformation [...] et que l'Art, une fois de plus, se transplantera vers l'ouest, à la suite des hommes, pour refleurir dans les Amériques ${ }^{29}$. Le moment historique de revanche sur l'envahissement de la laideur dans le monde correspond à l'instant où les «décadents» se précipitent dans l'action et lutter pour l'avènement d'un monde débarrassé de la laideur ${ }^{30}$.

21 Il n'est pas difficile de saisir Henry Van de Velde lui-même derrière ce portrait-fantôme du « décadent ». Après la mort de sa mère, Van de Velde fut saisi en 1890-1891 d'une neurasthénie qui le tenait éloigné pendant plusieurs années des grandes villes. Pendant cette période s'amorçait sous la lecture des ouvrages de John Ruskin et de William Morris un tournant qui allait conduire Van de Velde à se rapprocher des arts décoratifs pour chasser la laideur du monde afin de le rendre vivable pour tous. La laideur, telle que la présentait Ruskin et Morris, était interprétée comme le reflet d'une injustice sociale qui sollicitait l'action de l'artiste. Les contours de la mission de Van de Velde se précisent donc: la lutte contre la Laideur se définit très exactement comme combat pour un « monde meilleur », esthétisme et socialisme vont de pair ${ }^{31}$.

\section{L'ornement, arme contre la laideur}

22 Le nom d'Henry Van de Velde continue d'être associé à l'idée de la « ligne comme force " (Die Linie ist eine Kraft ${ }^{32}$. Cette formule qui résume abusivement sa pensée, demande à être interrogée.

L'esthétique matérialiste de Van de Velde est influencée comme celle des néoimpressionnistes - dont il fut lui-même un des principaux représentants jusqu'à son "tournant» vers les arts décoratifs dans les années 1885 - par une conception scientiste de l'œuvre d'art qui associe à certaines couleurs et certaines formes de lignes une stimulation particulière sur l'appareil nerveux humain. Ainsi, par exemple, une ligne ascendante était considérée comme stimulant la bonne humeur, de même que la couleur rouge. Les théories de Chevreul, Ogden Rood, des savants allemands Helmholtz et Wundt étaient reçus par les artistes comme une légitimation de leurs propres démarches pour définir un art capable de jouer un rôle social.

24 Van de Velde était lui aussi convaincu qu'une "bonne » ligne était porteuse d'une certaine forme d'énergie vitale qu'elle transmettait à son public ; une "mauvaise » ligne en revanche privait son entourage d'énergie. La laideur est donc au sens premier « énervante »: elle prive celles et ceux qui doivent la supporter d'énergie, de vitalité, de joie de vivre.

Pour être utile, l'artiste doit donc donner au monde de «belles formes " qui correspondent aux constantes anthropologiques de l'homme, exprimés par les termes de «rythme » et de «sensibilité ». L'ornement prend dans cette perspective une importance majeure : il ne s'agit pas d'un agrément superflu accolé à la surface des objets utilitaires, mais d'une expression d'une sorte d'élan vital qui habite les objets bien conçus, et que Van de Velde désigne sous le nom générique de "force ». Le «nouvel ornement» pratiqué par Van de Velde se distingue par le fait de ne 
représenter que des «formes abstraites, qui sont fondées sur le rythme, comme ce fut déjà le cas pour l'ornement grec $^{33}$.»

$\mathrm{Du}$ coup, il faut débarrasser l'ornement de toutes les réminiscences naturalistes, et éviter même la stylisation d'un objet qui pourrait être reconnu. A la différence par exemple du "style floral» de l'École de Nancy (Gallé, Guimard), qui recourait à des ornements dont les formes étaient inspirées par la faune et de la flore locales, Van de Velde insiste sur la nécessité de d'un ornement "pur, qui cherche en lui-même les éléments dont il est composé et qui invente des formes en puisant dans des moyens de construction nécessaires ou qui donnent vie à la matière inerte grâce à la répartition rythmique de la lumière et de l'ombre ${ }^{34}$.» En faisant coïncider la forme et la fonction et en évitant la stylisation de la nature, les Grecs ont découvert le moyen de conférer à leur architecture "la vie, tout comme le culte dionysiaque $»^{35}$. Van de Velde se targue d'avoir découvert la force principale de l'ornement: la «ligne complémentaire », qui agit un peu comme la "couleur complémentaire» des néo-impressionnistes pour renforcer la vitalité dégagée par la ligne: «Une ligne est une force, qui agit comme toutes les forces élémentaires : plusieurs lignes en relation les unes avec les autres mais allant dans des sens contraires ont le même effet que plusieurs forces élémentaires qui se heurtent les unes aux autres ${ }^{36} . »$ L'artiste peut ainsi conférer l'énergie qui habite en lui au monde qui l'entoure, il peut en faire cadeau à l'humanité : (la ligne) « emprunte son énergie à celui qui l'a tracé37».

Le mot d'ordre "la ligne est une force" est donc à situer dans le contexte d'un vitalisme fin de siècle, qui se croit légitimé par des avances ultérieures de la science. Van de Velde est convaincu de l'utilité sociale de son "invention»: l'ornement rationnel, produit à large échelle peut permettre à l'humanité de sortir de son état d'aliénation.

\section{Vers l'éradication industrielle du laid}

Dans un premier temps, l'action bénéfique des «belles formes » ne peut s'exercer que dans des espaces restreints, tant le monde est encore enfermé dans la laideur et incapable d'apprécier autre chose. Le Beau pénètre donc le monde du laid sous la forme des bibelots (Kleinkunst), que les décadents savent apprécier comme promesse d'un monde meilleur ${ }^{38}$. Sur ce point aussi, on pense involontairement à l'amour de Benjamin pour les petits objets, les bribes d'une culture déjà abandonnée, et qui recèlent selon lui une dimension d'utopie que le monde moderne a chassée ${ }^{39}$.

À partir des bibelots il devient possible de créer des formes plus importantes. Van de Velde en viendra à prendre ses distance avec Ruskin et Morris, auxquels il reproche, sur un ton de plus en plus véhément, leur opposition à l'âge des machines et la nostalgie d'un Moyen âge dans lequel « ils reconnaissaient tous les deux, des conditions d'harmonie et de lien entre l'humanité d'alors et les œuvres de l'architecture et des arts pratiqués par les artisans $»^{40}$.

Henry Van de Velde était convaincu, comme les cercles anarchistes auxquels il appartenait, que l'avenir de l'humanité pouvait être radicalement changé en peu de temps, grâce à une utilisation « rationnelle » de l'industrie. Tel son ami Paul Signac qui montrait dans sa fresque Au temps d'harmonie, dont le sous titre n'était autre que «L'âge d'or n'est pas dans le passé, il est dans l'avenir » une humanité réconciliée avec ellemême jouissant de son existence dans un cadre digne de Virgile ${ }^{41}$, Van de Velde lui 
aussi était convaincu que l'industrie pouvait libérer l'homme, et le placer dans une nature vierge.

31 Selon le scénario imaginé par le créateur belge, l'industrie devait être confié à des hommes sages (on pense immédiatement à un gouvernement d'artistes-philosophes, tel que le souhaitait Nietzsche) pour lutter de toutes les forces contre la laideur. Là où Ruskin et Morris avaient eu le tort de souhaiter le retour vers un Moyen Âge préindustriel, Van de Velde plaide pour une éradication industrielle de l'éclectisme ${ }^{42}$.

Les prémices de l'âge d'or sont déjà visibles : ce sont les constructions en acier, comme le pont du Forth of Firth ou la Tour Eiffel ${ }^{43}$. Dans ces constructions d'une "sévérité dorienne " (Van de Velde) aucune "sentimentalité » n'entrave la création rationnelle. Ces constructions sont comme un avertissement adressé au «monstre » de la laideur, que les chevaliers de la cause du Beau vont défier avec cette arme formidable qu'est l'industrie :

J'ai vu l'art se bardant à la façon légendaire et défiant le monstre et quand le sang noir et corrompu se répandra, l'hosanna montera au ciel et des fumées des hautes usines se déploieront insensiblement comme les grandes ailes blanches des anges annonciateurs ${ }^{44}$.

33 Ce « défi au monstre de la laideur » lancé pour la première fois en français en 1890, et repris ultérieurement en dans la collection d'essais Kunstgewerbliche Laienpredigten (1902) ne s'est pas concrétisé. Dressant en 1906 le bilan de la grande exposition d'art décoratif de Dresde, Van de Velde se montre déçu par l'évolution d'un style nouveau qui n'en finit pas d'arriver, voire par les tendances «réactionnaires" qui se manifestent dans le retour à un style néo-Biedermeier tel que le pratiquent de créateurs tels que Richard Riemerschmid. Donc un nouvel historisme! Même si Van de Velde est prêt à admettre que cette "prudence » est dictée par la «bonne foi » des artistes, il n'en voit pas moins une régression.

Mais Van de Velde n'arrive pas non plus à se familiariser avec un style totalement épuré, tel que le pratiquera le Bauhaus, ou Le Corbusier - dans son œuvre abondante, on ne relève aucune remarque sur ces deux mouvements, et ce n'est certes pas un hasard. Il est donc à notre avis abusif de tirer une ligne directe entre l'École d'arts décoratifs (Kunstgewerbliches Seminar) dirigée à Weimar par Van de Velde entre 1902 et 1914, et le Bauhaus. Il est vrai que Van de Velde a inclus dans la liste de ses trois successeurs possibles aussi le nom de Walter Gropius, dont il connaissait certainement les FAGUS-Werke à Ahlfeldt an der Leine (1910), qui anticipent le style que nous associons au Bauhaus : rideau de fenêtres et toit plat. Il nous semble pourtant que cette décision était dictée par d'autres motivations qu'un accord esthétique : à un moment où il était critiqué de toutes parts pour sa non-appartenance à la nation allemande, Van de Velde voulait mettre son École à l'abri de la fermeture (qui arriva pourtant) en recrutant une personnalité dynamique dont il avait pu déceler les qualités de leadership au sein du Werkbund.

\section{Pour une moderne apollinienne}

Le cas Van de Velde offre le rare privilège d'une critique de la modernité à partir d'une position elle-même moderne: rien de plus éloigné de sa position que les thèses chauvinistes et rétrogrades d'un Hermann Langbehn qui dans son ouvrage Der Rembranddeutsche (1890) voulait faire revenir l'Allemagne à l'âme germanique 
qu'incarnerait le maître d'Amsterdam, ou les tenants du Bund Heimatschutz (Association pour la protection du paysage national) qui souhaitaient revenir aux formes générées par le «sol » et la « race» de ceux qui l'occupaient. Mais Van de Velde n'est pas non plus le représentant d'un fonctionnalisme intégral comme le prônent certaines parties du Werkbund, dont il fut pourtant l'un des membres les plus célèbres. Et par son plaidoyer pour un "ornement rationnel», il offrait le flanc au plus célèbre des fondateurs d'une modernité épurée, Adolf Loos. Ainsi, Van de Velde est non seulement un artiste entre les patries, mais aussi un théoricien qui sur le champ de bataille des "modernes" se trouve entre toutes les lignes de front, au point de remettre en question la notion même d'avant-garde.

En effet, Van de Velde apparait comme l'un des représentants d'un courant important de l'histoire de l'art de la fin du XIX $x^{e}$ siècle, placé sous le signe de ce que le germaniste Gerhard Neumann désigne comme le "phantasme grec $»^{45} 44$. Il s'agit d'un courant représenté par des personnalités telles que Walter Rathenau, le comte Harry Kessler, Rudolf Alexander Schröder et de nombreuses autres représentants d'une génération née aux alentour des années 1860, et profondément influencé par les enseignements de Friedrich Nietzsche ${ }^{46}$. Dans ses écrits théoriques, Van de Velde ne cesse de faire référence à la Grèce présocratique, qu'il considère comme un modèle pour les temps à venir. Expurger de la pierre des «formes mortes » qui la hantent depuis l'avènement du christianisme, voilà comment pourrait se résumer son Kunstwollen. Le retour grâce au "Style nouveau » à une "unité stylistique dans toutes les expressions de la vie", réclamé par Nietzsche dans la Deuxième considération inactuelle par référence explicite à la Grèce ancienne, l'identité postulée entre "Laideur » et " déchéance morale », ce sont là autant de signes qui attestent la prégnance d'une esthétique classique, placée sous le signe du dieu de l'harmonie et de l'ordre: Apollon, et non pas sous celui de Dionysos, comme Van de Velde le prétend ${ }^{47}$. C'est toujours vers l'idée du temple grec que retourne l'imagination de Van de Velde: «Nous avons l'exemple sublime d'un monument d'abord naïf et indigent [...] mais que [...] le génie d'une race prédestinée pénétra de si magiques artifices [...] qu'il nous apparaît encore aujourd'hui que nous l'apercevons en ruine, - le Temple Grec ! - comme une création palpitante, se dressant devant nous [...] "infaillible, resplendissante, clairement définie et maîtresse d'ellemême" ${ }^{4}{ } » !$

\section{NOTES}

1. Je remercie Priska Schmickle von Minckwitz pour l'aide qu'elle a apporté à la rédaction de cet article. Le lecteur s'étonnera peut-être de l'orthographe changeante du nom d'Henry Van de Velde, due à son appartenance à plusieurs communautés de langue. Comme tous les artistes originaires des Flandres, il s'écrit avec un « V » majuscule en français (comme «Van Eyck» ou «Van Gogh ») et avec un « V » minuscule en allemand. Voir article « Velde, Henry Van de », in : E. Bénézit : Dictionnaire critique et documentaire des peintres sculpteurs dessinateurs et graveurs etc..., Paris, Gründ, 1999, t. 14, p. 101 et 102. Van de Velde écrivait en français, mais pour certains textes parus en allemand, la version originale a disparu. Plutôt que de « retraduire » Van de Velde dans 
son propre idiome, nous citons alors le texte allemand. Les écrits de Van de Velde sont mentionnés en abrégé avec l'année de leur publication ou réédition après leur première mention complète.

2. L'installation de Van de Velde en Suisse est due à la grande hostilité qui régnait dans la Belgique d'après-guerre à l'égard de tous ceux qui s'étaient mis au service de l'occupant allemand; Van de Velde avait occupé entre septembre 1940 et avril 1942 la fonction de «conseiller artistique » auprès du "Commissariat général de la Restauration du Pays ». Bien qu'officiellement blanchi, l'artiste dut partir en exil, à l'âge de quatre-vingt trois ans. Voir Henry Van de Velde: Les mémoires inachevées d'un artiste européen, texte établi et commenté par Léon Ploegaerts, Bruxelles, Académie Royale de Belgique, 1999, vol. 1, Les Mémoires, p. 505-529.

3. En Allemagne de l'Ouest le souvenir de Van de Velde fut notamment tenu vivant par Hans Curjel, qui avait édité un choix de ses œuvres théoriques (Henry van de Velde : Zum Neuen Stil, texte établi et commenté par Hans Curjel, Munich, Piper, 1955) et une version allemande, abrégée, de son autobiographie (Henry van de Velde. Geschichte meines Lebens, texte établi et commenté par Hans Curjel, Munich, Piper, 1962, Après sa disparition, un jeune architecte, KarlJürgen Sembach continuait à éclairer son œuvre en Allemagne de l'Ouest, tandis qu'en R.D.A. fut publiée en 1967 l'excellente thèse de Karl-Heinz Hüter : Henry van de Velde. Sein Werk bis zum Ende seiner Tätigkeit in Deutschland, Berlin, Akademie-Verlag (Schriften zur Kunstgeschichte, 9). À Hagen fut fondée une société Van de Velde, sous l'impulsion de Herta Hesse-Frielinghaus, la conservatrice du Musée Folkwang, fondé par Karl Ernst Osthaus, et dont l'aménagement intérieur fut confiée à l'architecte belge. (Le mécène Karl Ernst Osthaus avait été le premier biographe de l'artiste: Van de Velde. Leben und Schaffen des Künstlers, Hagen, Folkwang-Verlag, 1920). En Belgique, la monumentale œuvre d'Abraham Marie Hammacher Le monde d'Henry van de Velde (Anvers, Mercator, 1967) dominait une production par ailleurs très fragmentaire. Deux éditions concurrentes des «Mémoires" d'Henry Van de Velde existent actuellement: celle déjà mentionnée par Léon Ploegaerts, qui tient compte de toutes les variantes mais n'offre qu'un appareil de notes explicatives spartiate, celle éditée par Anne Van Loo, qui présente un texte unifié, mais accompagnée de notes abondantes (Henry Van de Velde : Récit de ma vie, 1, 1863-1900, 2. 1900-1914, texte établi et commenté par Anne Van Loo, Bruxelles et Paris, Versa/Flammarion, 1992 et 1996.

4. Pour la redécouverte de Van de Velde par le grand public, il faut mentionner le rôle de la grande rétrospective Henry Van de Velde, Ein europäischer Künstler in seiner Zeit organisée en 1992 à Hagen, Berlin et Anvers. Le catalogue de cette exposition, établi sous la direction de Klaus-Jürgen Sembach et de Birgit Schulte (Cologne, Wienand, 1992) constitue encore aujourd'hui une référence clé. À Berlin, le Musée Bröhan, consacré aux arts décoratifs, continue à mettre en valeur les belles créations de l'artiste dans sa collection, voir Ingeborg Becker : Henry van de Velde in Berlin, Berlin, Reimer, 1993 (rééd. 2004).

5. «... der Bäcker, der über die Form der "Weißbrötchen", der Kaufmann, der über die verschiedenen Behälter nachdenkt, in denen er Käse und Konserven zum Kauf anbietet, der Friseur, der sich den Sinn der Haartrachten und des Schnurrbartschnittes klar macht: sie alle arbeiten daran, die wesentlichen Formen alles dessen, was im Leben vorkommt, wiederzufinden. » In : Henry van de Velde : « Das Streben nach einem Stil, dessen Grundlagen auf vernünftiger, logischer Konzeption beruhen », in : id. : Vom neuen Stil : der « Laienpredigten » II. Teil, Leipzig, Insel, 1907, p. 21-83 et p. 32-33. Ce passage a été omis dans la version française de ce texte, parue en 1916/17 à Weimar sur presse privée, publiée dans le recueil Les Formules de la Beauté architectonique: parce que Van de Velde était lui-même un peu gêné d'un tel excès dans l'esthétisme?

6. Sur le parallélisme de la création artistique et de la réflexion théorique, voir l'introduction de Hans Curjel intitulé « Leben und Schriften » qui précède les textes choisis de l'ouvrage Henry Van de Velde: Zum Neuen Stil, op. cit., p. 5-22. Les écrits théoriques servaient aussi à faire passer au 
second plan un certain nombre de reproches qui lui étaient régulièrement adressées quant à la qualification nécessaire pour exercer son métier d'architecte. Peintre de formation, Van de Velde n'avait appris aucun des métiers habituellement associés à la fonction d'architecte : il ne savait ni faire des calculs statistiques, ni établir des plans de construction; ces tâches étaient confiées à des proches collaborateurs, notamment le Finlandais Sigurd Frosterus.

7. Nous nous référons bien sûr à Adolf Loos : Ornament und Verbrechen (1907).

8. Si nous nous bornons à la " première " période de Van de Velde, c'est que sa pensée théorique, sans se renier, connaît après 1920 des infléchissement majeurs dus au contact de la nouvelle génération d'architectes. La question de l'ornement constitue toujours un enjeu de réflexion théorique, mais sa pratique architecturale a pris un tournant l'amenant vers des formes plus compactes. Sans doute pour des raisons stratégiques et de tact, Van de Velde s'abstient de commentaires sur le Bauhaus, dont il se considère en quelque sorte le créateur indirect pour avoir proposé Walter Gropius à sa succession à Weimar. À l'occasion de l'Exposition Internationale des Arts Décoratifs à Paris en 1925, il consacre un ouvrage non dénué de sympathie à la nouvelle génération du design français, renouant avec son rôle de médiateur entre la France et l'Allemagne: Der Moderne Stil in Frankreich, Berlin, Wasmuth, 1925. Tout en se félicitant de l'avancée des créateurs français qui ont su ouvrir une voie indépendante de la sienne, il revient longuement dans la préface sur la question d'un «style pur ", atemporel, lié à la corporalité et au geste de l'homme, retrouvant ainsi une problématique esquissée dès ses premiers écrits (op. cit., p. 4,5 et 8$)$.

9. Voir Priska Schmückle von Minckwitz : L'ORNEMENT, un tapuscrit inédit de Henry Van de Velde zur l'origine de l'ornement et sur l'ornement structo-linéaire et dynamographique, DEA sous la direction de Bruno Foucart, Paris IV-Sorbonne, 2004.

10. Pour la fascination de Van de Velde pour le mouvement, voir sa «déclaration d'amour » in Amo « [...] c'est dans l'action que s'exalte au plus haut degré la beauté des corps humains, et pour définir la beauté d'un corps d'homme qui lutte ou qui boxe, ou d'un corps de femme emportée par la danse, il n'y a plus de mots et plus d'images. » Formules de la Beauté architectonique (1978), p. 39.

11. Cf. Hippolyte Taine: Philosophie de l'art (1865); nous ne pouvons approfondir ici l'histoire compliquée de la réception de Taine, revendiquée aussi bien par les " positivistes » que par les «idéalistes », cf. Pierre Desan: "Taine, positiviste ou idéaliste?», in: Dialogue, 21/1982, p. 661-669.

12. «Prinzipielle Erklärungen» in: Kunstgewerbliche Laienpredigten (1902), p.137-195, p. 147. Rappelons que Taine avait fait une exception dans sa présentation de l'histoire de l'art, en soulignant dans la cinquième partie de sa Philosophie de l'art, le caractère " universel » de l'art grec. Tel est aussi le but de Van de Velde.

13. Ibid., p. 187 et 188.

14. Il faut entendre "psychologie » dans ce cas dans un sens pré-freudien, c. à d. comme théorie des stimulations s'appliquant sur notre système sensoriel et nerveux.

15. «Depuis quatre siècles, depuis l'accomplissement de l'art gothique, l'arbre est mort qui s'élevait en nos contrées, marquant là une étape de quelques siècles dans cette marche de l'art, de l'est à l'ouest, à l'extrême limite duquel sa destinée doit s'accomplir » [...] Or, la dépravation des hommes était telle qu'ils dénommèrent "Renaissance » cette désagrégation impie. Et c'est l'ère des tableaux et des statues et des objets d'art, qui ne sont plus un apport soumis à l'idée de l'ornementation une et même. Chacun d'eux se limita vaniteusement à sa seule et précaire raison d'être. Et lors le tableau et la statue se dépouillèrent de toute vertu ornementale et essentielle, se firent anecdotique, sans plus, et pénétrèrent pour la première fois dans les demeures. » Henry Van de Velde : "Déblaiement d'art », in : Henry Van de Velde : Déblaiement d'art, suivi de La Triple offense à la Beauté, Le Nouveau, Max Elskamp, La voie sacrée, La colonne, Bruxelles, Editions des Archives d'Architecture moderne, s.d. (1979), p. 7 et 8. La condamnation de la Renaissance 
comme début d'une décadence de l'art est un thème répandu dans tout le dix-neuvième siècle, et se trouve aussi bien chez les peintres nazaréens allemands que chez les préraphaélites anglais. Dans sa condamnation de la Renaissance, Van de Velde se réfère notamment à William Morris, lui-même étroitement lié à William Burne-Jones, l'une des principales personnalités des Préraphaélites, voir « William Morris », in Kunstgewerbliche Laienpredigten (1902), p. 104-106.

16. «Durch die französische Revolution wurde urplötzlich eine langjährige Entwicklung des Geschmacks und des Stils, die der Renaissance ihren Stempel aufgedrückt hatte, abgebrochen. Nach der Revolution beginnt eine unheilvolle Ära, während der die Menschheit allmählich den Geschmack und den Sinn für Schönheit und für Stilgefühl verliert. ", "Vorrede », in : Vom neuen Stil : der « Laienpredigten» II. Teil, Leipzig, Insel, 1907, p. 1-3, p. 1.

17. «Eine Epoche ohne Stil ist ein Schiff ohne Steuer, das den Winden preisgegeben ist. Wenn es trotzdem einen Hafen erreicht, so ist es der Hafen des Zufalls, wo es höchstens den erlittenen Seeschaden reparieren kann» in: Henry Van de Velde: "Die veränderten Grundlagen des Kunstgewerbes seit der französischen Revolution », in : Vom Neuen Stil (1907) p. 4-20, p. 4.

18. Voir Walter Benjamin : Berliner Kindheit um 1900 (1932) in : id.: Gesammelte Schriften, texte établi et commenté par Rolf Tiedemann et Hermann Schweppenhäuser, Francfort/M Suhrkamp 1980, t. 10. Faute de place, nous ne pouvons approfondir ici la parenté entre Walter Benjamin et Henry Van de Velde. Ce sera l'objet d'un prochain article, à paraître in : Gérard Laudin (sous la dir.) : Berlin, Paris 2006.

19. Déblaiement, p. 9 et 10.

20. Cf. sa critique de William Morris : «Eine allgemeine, durch eine hauptsächlich religiöse Empfindung verursachte Verstörung macht uns mehr empfindlich für moralische Häßlichkeit als für die materielle. Die Wirkung des christlichen Glaubens und seiner Lehre [...] zerschmettert mit großen Worten, mit pompösen Sprüchen alle Lebenskeime, die in uns sich regen und die heute unser Stolz sind. » in : «William Morris », in : Kunstgewerbliche Laienpredigten (1902), p. 89.

21. P. ex. Formules de la Beauté architectonique (1978), p. 8.

22. Cf. "Eine Predigt an die Jugend" in: Kunstgewerbliche Laienpredigten (1902), p. 55 ; «Die schlechtesten Gemälde, die schlechtesten Skulpturen hatten jene erbärmliche Geistesrichtung am meisten befriedigt, und wie jene, die das größte Quantum Sentimentalität besaßen, die größte Anziehungskraft auf die Herzen der Menschen ausübten, wird die Sentimentalität noch so lange dauern, als bis das Bedürfnis nach Bildern und Skulpturen fast vollständig geschwunden sein wird. » cf. aussi Die drei Sünden wider die Schönheit, p. 31 : « [...] das war das Programm, das, nach meinem Gefühl, mit der Hässlichkeit fertig werden und dem Uebel Einhalt tun sollte, all diesem Unheil, entstanden durch die E i n m i s c h u n g d e r P h a n t a s i e in Bezirken, wo sie nichts zu tun hatte. » Plus loin, Van de Velde se réfère explicitement à Platon: "wir sind nun dahin gekommen, nach Platons Auffassung kundzutun, daß der wesentliche Charakter der architektonischen Schönheit der ist, was er immer gewesen ist: die vollkommene Übereinstimmung der Mittel mit dem Zweck. » Ibid., p. 175.

23. «Die Vernunft und ihre Schöpfungen : die Logik sind diese wenigen Prinzipien, aus denen die alten Stile entstanden sind, und darauf wollen auch wir den Stil unserer Zeit begründen. » Ibid., p. 142.

24. «Et la nouvelle atmosphère créée par les idées générales modernes et par la transformation profonde, organique des façons de satisfaire les besoins de la vie matérielle, ont amené la naissance d'un nouveau type d'Homme, si différent de celui de la première moitié du siècle dernier que l'on me permettra bien de distinguer ainsi l'un de l'autre : l'Homme moderne et l'Homme prémoderne ! [...] Il serait frappant d'évoquer toute la série des objets qui fournissent à l'homme le moyen de se soigner (etc...) aux objets destinés à satisfaire les même besoins de l'homme prémoderne » in : Formules de la Beauté architectonique (1978), p. 15.

25. Ibid., p. 17.

26. Ibid., p. 11 . 
27. Ibid., p. 5.

28. Déblaiement (1979), p. 14.

29. Ibid., p. 14.

30. «Or voici qu'une classe parmi les hommes, dont le coeur est resté intact comme leurs mains de tout partage de l'or qui nous a souillé tous - je veux dire le Peuple - va recueillir l'Art [...] et, quand ce reconnurent ceux-là qu'une rancœur infinie des choses, une gêne insurmontable dans le commerce avec les hommes confinaient de plus en plus au sommet des Tours, ils sacrifièrent joyeusement leur cher isolement et se rendirent aux «Maisons du Peuple». Et les décadent devinrent des militants. » in : Déblaiement, p. 23.

31. Cf. aussi « William Morris », p. 120 et p. 132.

32. «Prinzipielle Erklärungen », p. 188.

33. Je cite le passage de manière intégrale: "Die Faktoren, die auf die Abänderung des Ornaments gewirkt haben, sind empfindsamerer Art als die, welche in der Art des Bauens tätig geworden sind, und wenn ich später von einem neuen Ornament reden werde, dem das Prinzip der Logik und der Deduktion nicht mehr wie früher fremd sein wird, müssen sie sich nichts anderes darunter vorstellen als das, was der Wirklichkeit entspricht : nämlich, daß das Bedürfnis, logisch und vernünftig zu sein, neuerdings ein Gebiet betreten hat, das bis dahin einzig der Gefühlsrichtung und der Befriedigung des Sinns für Äußerlichkeiten vorbehalten war, sei es, daß die Ornamentik unserem Empfindsamkeitsbedürfnis Genüge tat durch natürliche Elemente, welche sie bald als Symbole, bald als wirkliche Gegenstände dem Bereiche der Menschen, Tiere oder Blumen entnahm, sei es, daß sie unseren Gesichtssinn durch abstrakte Formen befriedigte, deren Kernpunkt der Rhythmus war, wie es auch bei der griechischen Ornamentik der Fall gewesen ist. » Ibid., p. 149.

34. (die reine Ornamentik) « welche ihre Bestandteile aus sich selbst schöpft und Formen schafft, indem sie sich auf die notwendigen, nötigen Konstruktionsmittel stützt oder der sonst toten Materie Leben gibt, mittels Licht und Schatten, die hervorruft und rhythmisch verteilt. » Ibid., p. 183.

35. «Bei den Griechen ist das Ornament das Leben, es hat das Leben in sich, gerade wie der Dionysos-Kultus selbst. » Ibid., p. 185.

36. «Eine Linie ist eine Kraft, die ähnlich wie alle elementaren Kräfte tätig ist; mehrere in Verbindung gebrachte, sich aber widerstrebende Linien bewirken dasselbe, wie mehrere gegeneinander wirkende Kräfte. » Ibid, p. 188, ainsi que p. 191 et p. 192. Voir aussi Henry Van de Velde : « Die Linie » in : Die Zukunft 1902.

37. «Wenn ich nun sage, daß eine Linie eine Kraft ist, behaupte ich nur etwas durchaus Tatsächliches; sie entlehnt ihre Kraft der Energie dessen, der sie gezogen hat. » Prinzipielle Erklärung, p. 189.

38. «[...] il advint ainsi à toutes les époques de décadence que l'objet d'art, le bibelot furent particulièrement choyés, sans plus et pour eux-mêmes. [...] ils (les «décadents", AK) affectionnent l'objet d'art en lequel il leur est permis de reconnaître les complexités admirables et les entortillements de leur esprit [...] » in : Déblaiement (1979), p. 13.

39. Cf. Notamment WB: Einbahnstrasse (1928) in: id: Gesammelte Schriften, texte établi et commenté par Rolf Schweppenhäuser et Hermann Tiedemann, Francfort/M : Suhrkamp 1980, t. 10. Notons cependant que Benjamin, à la différence de Van de Velde est attiré, et non pas repoussé, par tous les objets qui sont remplies d'une «vie morte » et nous paraissent « laids » et insignifiants.

40. Formules (1978), p. 6.

41. Paul Signac: Au temps d'harmonie (L'Âge d'or n'est pas dans le passé, il est dans l'avenir), 1893-1895, huile sur toile, $300 \times 400 \mathrm{~cm}$, Mairie de Montreuil. La fresque était initialement prévue pour la Maison du peuple de Bruxelles, construite par Victor Horta (achevée en 1899).

42. « La Triple offense à la Beauté », in : Déblaiement (1979), p. 31-68, particulièrement p. 31-51. 
43. «Or, précisément à l'époque, où nous commençâmes à prêcher l'évangile d'un Style nouveau nous fûmes mis en présence de créations nous apparaissant, dans le frappant dépouillement de toute superfétation ornementale, dans la frappante nudité d'une construction rationnelle et conséquente; je veux parler de tous les édifices et de toutes les constructions à charpentes métalliques : les gares, les halls, les ponts ! [...] Pour la première fois, depuis longtemps, nous étions en présence d'éléments d'architecture organiques et dépouillés de tout ce qui pouvait induire à nous méprendre sur leur fonction. » Formules de la beauté architectonique (1978), p. 9.

44. Déblaiement (1979), p. 25.

45. Cf. Gerhard Neumann et Gerhard Schnitzler (dirs.) : Harry Graf Kessler. Ein Wegbereiter der Moderne, Freiburg i. B., Rombach, 1997 (Rombach Litterae, vol. 39).

46. Voir Alexandre Kostka: "Nietzsche - un penseur d'avant-garde comme enjeu intergénérationnel dans la revue 'Pan' (1895-1900)», in : Marc Cluet (dir.) : Le Culte de la jeunesse et de l'enfance en Allemagne 1870-1933, Rennes, Presses Universitaires de Rennes, 2003, p. 37-54.

47. Cf. note 34 .

48. Formules de la Beauté architectonique (1978), p. 18.

\section{RÉSUMÉS}

Le XIX siècle était une époque "croyante ", au sens le plus profond du terme. La pauvreté, les carences politiques - tout paraissait pouvoir être guéri : même la laideur. Parmi les nombreux apôtres de la Beauté, l'architecte et dessinateur Henry Van de Velde (1863-1957) se distingue par son radicalisme. Initialement inspiré par le mouvement "Arts and Crafts", qui, venu d'Angleterre, était parti à la conquête du continent, le peintre belge se détournait de la peinture de chevalet et développait une synthèse des «beaux arts » et des " arts décoratifs ». Il attachait une importance particulière à un dessin expressif de la ligne, ce qui lui a valu la réputation douteuse d'avoir inventé le «style ténia ». Les succès à sensation de ses expositions à partir des années 1890 reposaient cependant sur un malentendu, que Van de Velde entendait éclaircir dans ses écrits théoriques - sans y parvenir. L'« ornement rationnel », qui ne se réfère pas à des motifs empruntés à la nature, mais à l'expressivité spontanée de la gestuelle et de la réceptivité de l'homme, devait permettre la guérison d'une crise de la modernité, dont l'origine remonte selon Van de Velde à la Renaissance.

Das 19. Jahrhundert war ein im tieferen Sinne «gläubiges» Zeitalter. Armut, politische Mißstände - alles schien heilbar: auch die Häßlichkeit. Unter den vielen Aposteln der Schönheit sticht der Architekt und Designer Henry van de Velde (1863-1957) durch seine Radikalität hervor. Anfänglich inspiriert von der Arts and Crafts Bewegung die am Ende der 1880er Jahre aus England her den Kontinent erobert, kehrt sich der belgische Maler vom Tafelbild ab und entwickelt eine Verquickung von «bildender» und «dekorativer» Kunst. Besonderen Wert legte er auf eine expressive Linienführung, die ihm den zweifelhaften Ruf des Erfinders des «Bandwurmstils» eintrug. Die Sensationserfolge seiner Ausstellungen seit Mitte der 1890er Jahre waren jedoch ein Mißverständnis, welches van de Velde in seinen theoretischen Schriften aufzuklären sucht - ohne Erfolg. Das «vernunftgemäße Ornament», welches sich nicht auf aus der Natur entlehnte Motive bezieht, sondern auf die spontane Expressivität menschlicher Gestik und Rezeptivität, sollte die Genesung einer Krise der Moderne ermöglichen, deren Genealogie van de Velde auf die Renaissance zurückführt. 
INDEX

Mots-clés : architecture, beauté, laideur

\section{AUTEURS}

\section{ALEXANDRE KOSTKA}

Université Cergy-Pontoise 